\title{
Telemonitoring: use in the management of hypertension
}

\author{
This article was published in the following Dove Press journal: \\ Vascular Health and Risk Management \\ 10 April 2014 \\ Number of times this article has been viewed
}

\section{Darshi Sivakumaran' Kenneth Anthony Earle ${ }^{1,2}$ \\ 'Thomas Addison Unit, St George's Hospital, London, UK; ${ }^{2}$ Clinical Sciences, St George's University of London, London, UK}

\begin{abstract}
Hypertension is a major modifiable risk factor for cardiovascular, retinal, and kidney disease. In the past decade, attainment rates of treatment targets for blood pressure control in the UK and US have increased; however, $<11 \%$ of adult men and women have achieved adequate blood pressure control. Technological advances in blood pressure measurement and data transmission may improve the capture of information but also alter the relationship between the patient and the provider of care. Telemonitoring systems can be used to manage patients with hypertension, and have the ability to enable best-practice decisions more consistently. The improvement in choice for patients as to where and who manages their hypertension, as well as better adherence to treatment, are potential benefits. An evidence base is growing that shows that telemonitoring can be more effective than usual care in improving attainment rates of goal blood pressure in the short-to-medium term. In addition, studies are in progress to assess whether this technology could be a part of the solution to address the health care needs of an aging population and improve access for those suffering health inequalities. The variation in methods and systems used in these studies make generalizability to the general hypertension population difficult. Concerns over the reliability of technology, impact on patient quality of life, longer-term utility and cost-benefit analyses all need to be investigated further if wider adoption is to occur.
\end{abstract}

Keywords: telehealth, health surveillance, virtual-led clinic

\section{Introduction}

Hypertension is a major, modifiable risk factor for cardiovascular disease (CVD), which is a leading cause of mortality in Westernized countries. The American Heart Association 2012 update reports mortality rates due to CVD per 100,000 men and women aged 35-74 years of 256 and 130 in the US, compared with 202 and 88 in the UK, respectively. ${ }^{1}$ The 2011 Health Survey for England suggests that hypertension affects $31 \%$ of males and $28 \%$ of females over the age of 16 , and that every $2 \mathrm{mmHg}$ rise in blood pressure (BP) is associated with a $10 \%$ and $7 \%$ increase in mortality risk from ischemic heart disease and cerebrovascular disease, respectively. ${ }^{2}$ Hypertension associated with diabetes is also a leading cause of retinal disease, chronic kidney disease, and end-stage renal failure in the Western world. ${ }^{3-5}$ Over the decade up to 2008, data from the US National Health and Nutrition Examination Survey showed that the prevalence of hypertension remained constant at around $30 \%$, as it did in the UK. ${ }^{2,6}$

The National Institute for Health and Care Excellence (NICE) in the UK defines hypertension in stages. Stage 1 hypertension is a clinic BP $\geq 140 / 90 \mathrm{mmHg}$ and subsequent ambulatory BP monitoring (ABPM) daytime average or average home
Correspondence: Kenneth Anthony Earle Thomas Addison Unit, St George's Hospital, Blackshaw Road, Tooting, London SWI7 ORE, UK

Tel +44208725 3902

Fax +44208725 I353

Email kearle@sgul.ac.uk 
BP monitoring (HBPM) of $\geq 135 / 85 \mathrm{mmHg}$. Treatment is advocated for all affected patients under the age of 80 years with any target-organ damage, established CVD, diabetes, renal disease, or a 10 year cardiovascular risk $>20 \%$. Patients without target-organ damage should be given lifestyle advice and have arrangements made for future BP monitoring. Stage 2 hypertension is a clinic BP of $\geq 160 / 100 \mathrm{mmHg}$ and subsequent ABPM daytime average or HBPM $\geq 150 / 95 \mathrm{mmHg}$. Severe hypertension is a clinic systolic BP (SBP) of $\geq 180 \mathrm{mmHg}$ and/or diastolic $\mathrm{BP}$ (DBP) of $\geq 110 \mathrm{mmHg}$, which should be treated regardless of age, comorbidities, and cardiovascular risk. Target BP is suggested by NICE as $<140 / 90$ in those under the age of 80 years, and $<150 / 90 \mathrm{mmHg}$ for those above the age of 80 years. $^{7}$

A recent study suggests that less stringent BP targets may be required in patients with diabetes. ${ }^{8}$ This opinion is related to the so-called J-shaped curve, which indicates that lower BP levels may be detrimental in some groups of patients. Therefore, especially in the presence of such comorbidities as diabetes and renal disease, it is important to be able to personalize or tailor treatment to the individual. There is a considerable evidence base showing the efficacy of BPlowering treatment in reducing the risk of CVD events in patients below and above 65 years of age. ${ }^{9}$ The risk reduction in stroke and coronary artery disease and delaying the progression of renal disease is of the order of $25 \%-35 \% .^{10-12}$ Encouragingly, recent data have shown that the proportion of patients aware of their hypertension, receiving treatment, and reaching goal BP has been increasing over the past decade in the US and UK. ${ }^{3,4}$ There is still a considerable level of improvement required for these outcomes. As shown in the 2011 Health Survey for England, 43.1\% of all hypertensive patients are currently not receiving medical therapy, and of those receiving treatment, $38.1 \%$ are inadequately controlled, with only $11 \%$ of men and $10 \%$ of women achieving their target $\mathrm{BP}$ of $<140 / 90 \mathrm{mmHg}{ }^{3}$

\section{Monitoring blood pressure}

The measurement of BP is one of the most common and fundamental procedures in medical practice. The process has evolved over more than 130 years, and has not been immune from the modern revolution in information technology.

Von Basch developed the first design for noninvasive BP monitoring in approximately 1881 . He utilized a waterfilled rubber bulb attached to a mercury manometer and determined SBP to be the point at which the arterial pulse was obliterated. In 1896, the concept of inflating a cuff placed around the arm to generate more uniform pressure on the brachial artery was developed by Scipione RivaRocci. Subsequently, clinicians observed the pattern of oscillations in the mercury manometers to estimate SBP and DBP, and in 1905 Korotkoff introduced auscultatory BP measurement. ${ }^{13}$ The auscultatory technique with the mercury sphygmomanometer is the most established method of noninvasive BP monitoring, upon which the majority of clinical trial outcomes are based.

Alternatives to using the mercury sphygmomanometer have been assessed and are considered able to improve accessibility of the measurement outside the clinical setting. The aneroid meter has a numbered dial, the cuff is manually inflated and blood pressure is measured using the auscultatory technique. ${ }^{14}$ Ma et al compared BP measurements from both mercury and aneroid sphygmomanometers and found no differences in readings from nearly 1,000 patients across 24 centers. ${ }^{15}$ However, the dexterity involved in using the aneroid meter and need for regular recalibration limits its role in HBPM.

The oscillometric technique overcame the need to detect the Korotkoff sounds, and is used in most digital meters. In this method, the mean arterial pressure is determined and systolic and diastolic blood pressures are deduced using preset algorithms. The measurements of these digital monitors have been shown to be comparable to invasive and noninvasive auscultatory techniques, and because of their ease of use and accuracy, they are employed for HBPM and ABPM. ${ }^{16}$

The diagnosis and management of hypertension usually takes place in a primary-care setting. There are several disadvantages of this approach, including the snapshot BP measurement, observer bias, and white-coat effect. Evidence has emerged for the role of ABPM and HBPM to identify and diagnose hypertension more accurately, and features in current guidelines. ${ }^{2}$ The accuracy and convenience of digital measurements has also allowed more BP profiling for individual patients. Furthermore, a recent meta-analysis suggests that HBPM is more effective but more costly than office/clinic BP measurements in achieving target BP. ${ }^{17}$

The incorporation of telemonitoring with BP measurement via wired and wireless technologies provides a method of reducing reporting bias. This sophistication of reporting could allow telehealth technologies to dramatically change the relationship between the patient and the health care provider in the management of hypertension. Therefore, it is necessary to assess the impact of the tech- 
nology itself and the patient's response to it as part of their health management.

\section{Telehealth: changing the delivery and impact of health surveillance}

Telehealth can be defined as the delivery of health-related services over distance using telecommunications. This could simply be a telephone conversation between a patient and health professional or the more complicated delivery of a procedure using robotic technology. Unlike the focus on treatment that characterized telemedicine, telehealth tries to encompass a more holistic approach to health care in the sophistication of the technology to monitor outcomes, transmit information, and promote learning that will prevent deterioration in health. The advantages of telehealth include the assessment of data in real time, and when coupled with decision-making strategies can accelerate the delivery of best practice.

When compared with specialist health professionals, decision-support systems can be more consistent in the delivery of clinical decisions that guarantee treatment intensification. Our group developed an artificial intelligence system written in the PROforma language that contained expressions representing clinical criteria to influence and plan treatment based on the NICE guidelines to manage hypertension. ${ }^{18}$ Clinic BP data on 219 patients seen either in a nurse- (NLC) or a physician-led clinic (PLC) were uploaded to a database, which was then subjected to the decision system in a virtual setting (VLC). The variables for decisions were cardiovascular risk score estimated from the Joint British Societies Cardiovascular Risk Assessor, SBP, DBP, and use of first-line drugs (FLDs) for managing hypertension. The FLDs were classed as angiotensin-converting enzyme inhibitors or receptor blockers, beta-blockers, calcium-channel blockers, and thiazide diuretics, which were entered according to whether they were prescribed at the maximal dose.

Therapeutic decisions were coded as: $1=$ monitor only, $2=$ increase FLD, $3=$ start a new FLD, or $4=$ refer for secondline agent. Target SBP and DBP were $<130$ and $<80 \mathrm{mmHg}$, respectively. The patients seen in the NLC and PLC clinics had similar mean (standard deviation) age, SBP, DBP, and glycated hemoglobin (63.2 [12.8] versus [vs] 61.6 [13.5] years, $P=0.38 ; 144.9[19.4]$ vs $142.2[12.6] \mathrm{mmHg}, P=0.29$; $77.6[11.8]$ vs 78.0 [10.2] $\mathrm{mmHg}, P=0.79$; and $8.0 \%$ [1.8\%] vs $8.1 \%$ [1.5\%], $P=0.09$ ). All patients had a CVD score of $>20 \%$. There were no differences between the groups in the prescription of any class of FLD, second-line agent, or the proportion on a maximal dose within any class. Decisions to monitor only in the PLC group were significantly higher and treatment altering decisions 2, 3 and 4 significantly lower than in the NLC group (67.6 vs 32.8 and 10.8 vs 35.1 , 13.5 vs $21.6,8.1$ vs $10.4 ; P=0.0001)$. Decisions in VLC did not recommend "monitor only," but suggested decisions 2 and 3, respectively, for $81 \%$ and $19 \%$ of the PLC group. VLC decisions 1, 2, 3, and 4 were made for $15 \%, 63 \%, 19 \%$ and $3 \%$, respectively, of the NLC group. The kappa estimate for agreement with the artificial-intelligence decisions of VLC was 0.1 (poor) in the PLC group and 0.3 (fair) for the NLC group. ${ }^{19}$

Therefore, a system using artificial-intelligence system algorithms can consistently recognize the impact of CVD risk and is more likely to suggest a treatment modification than either nurses or physicians in face-to-face consultations with the patient. Use of such decision support could possibly improve outcomes, and incorporation of such evidencebased decision-making systems could further develop the HBPM potential of telehealth.

The role of telehealth in health care delivery is still being actively debated. There are some obvious issues related to the adoption of new technology, but what is less easy to assess is the impact the interrelationship with care providers and technology may have on health behavior. Telehealth technology has the ability to deconstruct the traditional medical model typified in the standard clinical consultation. Although this technology has the ability to transfer care closer to home, which may ostensibly be better for patients, there is concern over its long-term efficacy and impact on quality of life.

The architecture of a typical telehealth system is complex, with many links between the components at which problems may arise, including authentication, capture, transfer, interpretation, contextualization of data, and provision of an action plan (Figure 1). The replacement of the face-to-face, closed, and relatively confidential immediate communication in the traditional medical model is difficult to replicate in telemonitoring. A great deal of sophistication and reliability in the hardware and software is required to capture patient confidence. Moreover, the health care provider has a crucial role in assessing the data (preferably in real time) to maintain the safety of the patient. In addition, considerable efforts are necessary to ensure that patients' data remain secure and confidential when distributed around such a complex system. The ideal scenario in which to deploy these systems will depend on the local infrastructure and resources and the patient demographic.

The most important domain in which to devolve care is considered to be the home. A number of trials have now 


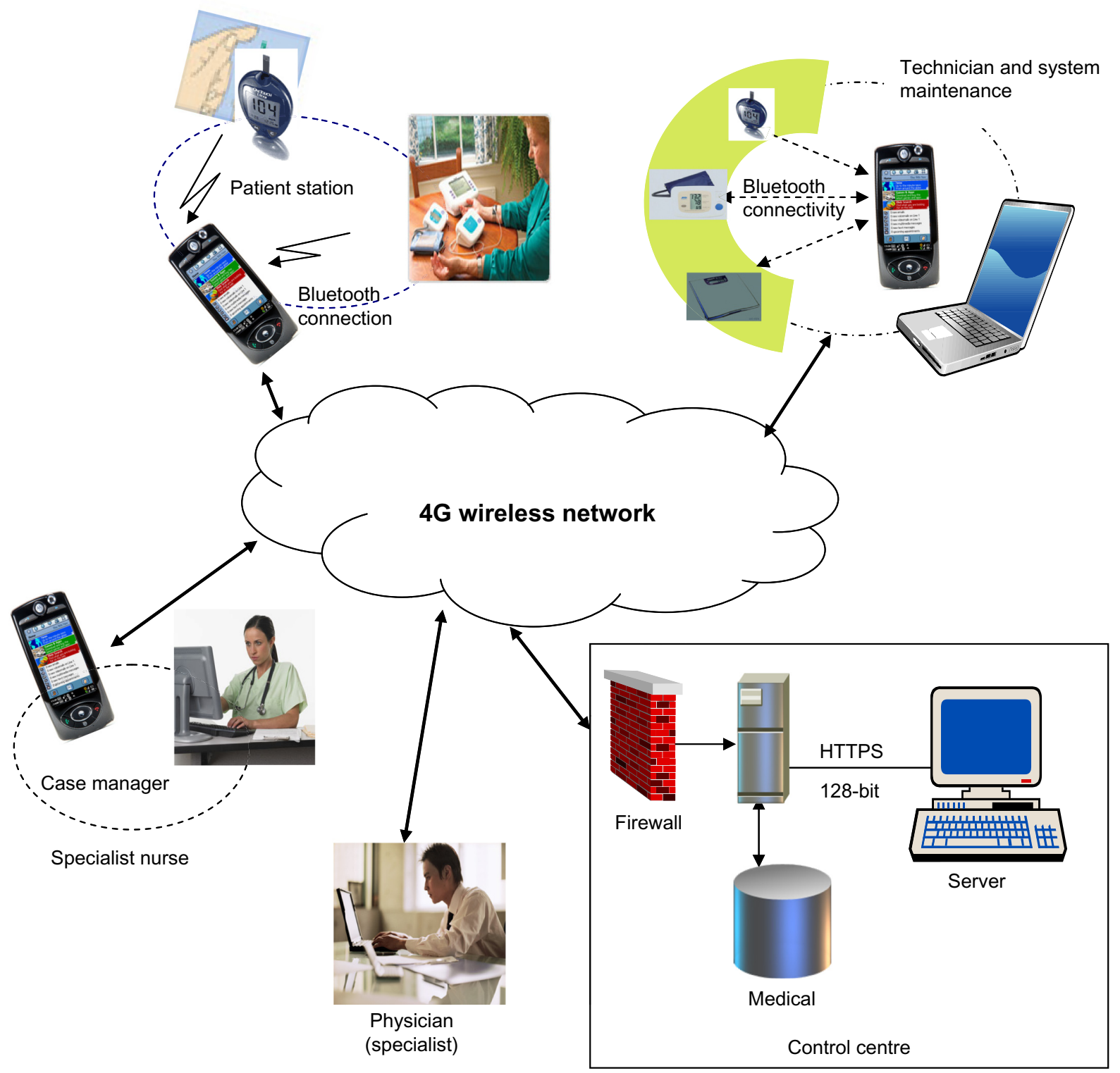

Figure I Schematic representation of a home (fixed) and smartphone (mobile) telemonitoring systems showing the key relationships between the patient technical and medical/nursing health professionals.

Notes: Reproduced by permission of SAGE Publications Ltd., London, Los Angeles, New Delhi, Singapore and Washington DC, from Istepanian RS, Zitouni K, Harry D, et al. Evaluation of a mobile phone telemonitoring system for glycaemic control in patients with diabetes. J Telemed Telecare. I5(3): I25-128. Copyright @ Sage Publications Ltd, 2009. ${ }^{35}$

been published trying to establish the clinical effectiveness of HBP telemonitoring (HBPT). However, there is a wide range of heterogeneity in the methodology in these trials, including variations in the technology of data transmission, measuring devices, frequency of monitoring, interface with different types of health care professionals to review and act on measurements, frequency of patient-to-health care provider contact, targets of $\mathrm{BP}$, and duration of study.

Omboni and Guarda carried out a recent meta-analysis of 23 randomized controlled trials of HBPT involving a total of 7,037 patients. ${ }^{20}$ Their analysis concluded that the HBPT showed a statistically significant improvement in SBP and DBP in comparison to usual care. SBP was reduced by $4.71 \mathrm{mmHg}$ (95\% confidence interval [CI] $6.18-3.24, P<0.001)$ and DBP by $2.45 \mathrm{mmHg}(95 \% \mathrm{CI}$ $3.33-1.57, P<0.001)$. A higher number of prescriptions for antihypertensive medications were issued in the HBPT group $(+0.40,95 \%$ CI $0.17-0.62 ; P<0.001)$, with a greater proportion of these patients achieving treatment-target BP (relative risk 1.16, 95\% CI 1.04-1.29; $P<0.001$ ).

McCant et al investigated the feasibility/technicalities of using HBPT in 588 patients with poor BP control randomized to three intervention groups $(n=441)$ using HBPT compared with 178 who received usual care. ${ }^{21}$ Patients in 
the intervention groups had to transmit their BP and pulse readings wirelessly to a home hub, which sent the data via the home telephone line to a server. Patients were required to check their BP every other day so that there would be a total of six readings in a 2 -week period. An automated alert process was put in place for technical problems and for when $\mathrm{BP}$ data were not sent according to the protocol. To resolve alerts, nurses made contact with the patients and provided any necessary intervention materials; the average resolution time for each alert was 4 days. After 6 months, only $75 \%$ of intervention patients were able to set up monitoring and adhere to the study protocol. A total of 693 technical alerts were generated by 267 patients, with $61 \%$ of all alerts being secondary to nonadherence. The 112 patients who generated more than two technical alerts were of a younger mean age (61 vs 64 years, $P<0.001)$ and more likely to be non-Caucasian ( $64 \%$ vs $47 \%, P<0.002$ ) compared to those that generated zero to two technical alerts. The device or system alerts included failure of the monitor to provide readings and problems with the telecommunications hub. Of note, the resolution time of the alerts was relatively slow and may have impacted patient confidence. Although further advances in telecommunications will allow some of these difficulties to be overcome, poor patient adherence (and confidence) can prove to be a significant limitation to the practical use of HBPT. Systems that involve a greater degree of patient-health professional interaction may offer greater cohesion and trust.

\section{Telehealth and the interface between medical and non-medical health professionals}

The reasons for the limited success in managing BP conventionally are complex. Some of the factors associated with the delivery of high-quality care and achievement of treatment goals relate to access, appropriateness of the pathway for monitoring of BP, patient compliance with treatment, and professional attitudes to the implementation and modification of treatment. Telemonitoring may have a role in overcoming the inertia in acting on BP readings, but itself does not have the benefit of the reasoned decision-making a trained physician has for not adjusting treatment. ${ }^{22}$ This emphasizes the importance of integrating this technology with the decisions made by practitioners who take account of the prevailing context in which BP was measured.

Pharmacists have been able to improve outcomes in hypertension management in the hospital setting, and increasingly are involved with care in the community. ${ }^{23} \mathrm{~A}$ systematic review of interventions to control BP concluded that systems with an organized review of treatment could produce a mean reduction in $\mathrm{BP}$ of $8.2 / 4.2 \mathrm{mmHg}$ and reduce all-cause mortality. ${ }^{24}$ Telemonitoring has the potential to link different health professionals together and integrate monitoring with prescriptive action.

A study by Green et $\mathrm{al}^{25}$ is of particular note with respect to the variation in delivering telehealth monitoring and management of hypertension. In this study of 778 patients, those in the active-intervention arms were asked to measure their $\mathrm{BP}$ with validated home BP monitors twice a week. One group worked with their physician, another used additional web-based resources to help manage their BP, and a third group used the web training plus pharmacist care. The trained pharmacist provided assistance with home BP monitoring and web communications via planned telecommunication. At the end of the trial, the proportion of patients achieving controlled BP $(<140 / 90 \mathrm{mmHg})$ was similar in those who received web training only compared with usual care $(36 \%$ [95\% CI 30\%-42\%] vs 31\% [95\% CI 25\%-37\%]; $P=0.21$ ). However, pharmacist-assisted, web-based HBPM significantly increased the percentage of patients with controlled BP (56\%, 95\% CI 49\%-62\%) compared with usual care $(P<0.001)$ and home BP monitoring and web training only $(P<0.001) .{ }^{25}$ In this population, the added interaction with a health care provider improved BP outcomes particularly in the group with higher BP, who were at greater risk of CVD. This study suggests that pharmacists have a key role in blood pressure management at the interface between the patient and technical advances in BP measurement.

\section{The patient-technology interface}

The Rosenstock health-belief model suggests that perceived seriousness of, and susceptibility to a condition with cues to action will improve an individual's readiness to change health behaviors or take up a health service. ${ }^{26} \mathrm{~A}$ key issue regarding the improved ability to monitor BP in different environments is to standardize the process to act on the outcome data and change medication and/or monitoring. In the Telemonitoring and Self-Management in the Control of Hypertension (TASMINH2) trial, 527 patients were randomized to usual care $(n=264)$ or HBPT with self-care $(n=263)$. Those in the intervention group were given preset instructions for two antihypertensive modifications they could make themselves if they had concerning BP readings on a number of occasions. At the end of the study, $80 \%$ of patients had completed the 12-month protocol, and data from 91\% were included in the final analysis. There was a significantly greater fall in 
mean SBP in the HBPT/self-care group compared to those in the usual care group at 6 and 12 months $(3.7 \mathrm{mmHg}, 95 \%$ CI $0.8-6.6 ; P=0.013)$ and $5.4 \mathrm{mmHg}(95 \%$ CI $2.4-8.5$, $P=0.0004)$, respectively. ${ }^{27}$ The study by Green et $\mathrm{al}^{25}$ and the TASMINH2 trial $^{27}$ emphasizes the importance of patient education, self-monitoring, and support for acting on advice provided with HBPT interventions. Therefore, in select groups, addressing the three concepts of Rosenstock et al's model could possibly improve the management of hypertension with telemonitoring.

Wireless communication such as Bluetooth technology may improve the patient experience with telemonitoring. It is more flexible than home-based systems, and approaches the ideal of delivering health monitoring for the patient anytime and anywhere in real time. Logan et al utilized HBPT using a validated, Bluetooth-enabled home BP device paired with a smartphone that ran an application that transmitted every reading to an application server. The data were then processed with decision rules, and an automated self-care message was generated and sent to the patient's smartphone following every reading. Examples of messages included reassurance that BP control was satisfactory, advice regarding the need for changes in the frequency of monitoring, or arranging follow-up with a health care professional. Nonadherence also triggered an automated voice message that was sent to the patient's phone line. In this study, 110 patients with uncontrolled systolic hypertension (and diabetes) were randomly allocated to either HBPT intervention and self-care support $(n=55)$ or usual care $(n=55)$. After 1 year, there was a significant decrease observed in the mean (standard deviation) daytime SBP within the intervention group of 9.1 (15.6) $\mathrm{mmHg}(P<0.0001)$, as well as between groups of $7.1(2.3) \mathrm{mmHg}(P<0.005)$. Treatment-target BPs were also achieved in a greater proportion of patients in the intervention than in the control group (51\% vs $31 \%, P<0.05)$. No significant change in BP was noted within the control group itself over the 12-month period. ${ }^{28}$ The improvement in BP in this study was independent of changes to antihypertensive medications or visits to physicians. In contrast, in the TASMINH2 study, 148 patients made at least one medication change and in the intervention group 0.46 (95\% CI 0.34-0.58) additional antihypertensive agents were used compared to the control group $(P=0.001)$. This difference was related to the difference in methodology, but together it shows that improvement in patient behaviors per se can bring about significant improvements in BP outcomes that can be facilitated by an appropriate telemonitoring system that also encourages self-care.
Patients may feel more isolated and become burdened by the process and responsibility of making, transmitting, and acting on these BP measurements. It has been suggested that telehealth may be potentially harmful and increase depression and anxiety. However, in a nested study in 1,573 patients with chronic pulmonary disease, heart failure, and diabetes, there were no statistically significant differences in anxiety and depression scores after 12 months in the telehealth group compared to those receiving usual care. ${ }^{29}$ In contrast, in the study by Green et al, those who had interaction with the health professional had better qualitative outcomes. ${ }^{25}$ In a meta-analysis of randomized control trials where quality of life could be assessed, it was found that physical health scores were significantly higher in HBPT $(+2.78,95 \%$ CI 1.15-4.41; $P<0.001)$, and there was no significant difference in mental health scores. ${ }^{20} \mathrm{~A}$ recent HBPT study comparing self-care and usual care alone found no significant differences in the anxiety component of the Hospital Anxiety and Depression Scale; however, within the intervention group there was a higher depression score at 12 months compared to their baseline. ${ }^{28}$ Although these data suggest telehealth does no harm, further qualitative analyses are required. In addition, these qualitative outcomes should be assessed when deploying telehealth interventions in groups with or at high risk of anxiety and depression, such as those with multiple comorbidities.

The clinical benefits of managing hypertension effectively in patients with other comorbidities complicating hypertension, such as diabetes, is well established..$^{30}$ However, data on the utility and efficacy of telemonitoring in populations at very high risk of CVD are limited. Our group performed the first randomized, controlled trial in patients with type 2 diabetes who were characterized by being at risk of progressive renal dysfunction. Both blood glucose and blood pressure and Bluetooth-enabled sensors were used together to transmit data to smartphones. We studied 137 patients who were randomized to either mobile telemonitoring $(n=72)$ or usual care $(n=65)$. Clinic BP was recorded at baseline and after 6 months. Patients in the intervention arm transmitted weekly to clinicians, who received the data in real time, and using a web-based application provided management advice to the patient and their physicians. In an intention-to-treat analysis, the study SBP fell significantly in patients in the intervention group (mean $[95 \% \mathrm{CI}]-6.5[-0.8$ to -12.2$] \mathrm{mmHg}$, $P=0.027)$ and remained unchanged in the control group $(2.1$ [9.3 to -5.0$] \mathrm{mmHg}, P=0.57$ ). Patients of African origin within the intervention arm seemed to benefit more from the intervention. In addition, those who achieved an SBP 
of $<120 \mathrm{mmHg}$ had lower average blood sugars than those with higher readings. ${ }^{31}$

In the Informatics for Diabetes Education and Telemedicine (IDEATel) study, ${ }^{32}$ 1,665 middle-aged Medicare recipients with diabetes were randomized to usual care or an intervention with a home telemedicine unit used for web access, videoconferencing with a health care professional and monitoring of glucose and BP. After 12 months, SBP and DBP were respectively lower in the intervention group $(3.4 \mathrm{mmHg}[P=0.001]$ and $1.9 \mathrm{mmHg}[P<0.001])$. Therefore, telehealth systems may benefit disadvantaged groups because of systematic health inequalities and ethnic or environmental factors that increase the susceptibility to poor disease outcomes. In this respect, the results of the ongoing study by Egede et al will be of interest. This group has planned a 4-year prospective, randomized clinical trial, which will test the effectiveness of technology-assisted case management in low-income rural adults with type 2 diabetes. Two hundred male and female participants, 18 years of age or older and with poor diabetes control will be randomized into the intervention arm using the FORA system - homebased telemonitoring using cable data transfer - coupled with nurse case management or usual care. ${ }^{33}$ The forthcoming Blood Pressure Control and Compliance to Treatment in Hypertensive Patients with Metabolic Syndrome: a Study Based on Home Blood Pressure Telemonitoring and Assessment of Psychological Determinants (TELEBPMET) trial aims to investigate HBPT vs usual care in patients with metabolic syndrome, where telehealth may have an application in patients who are at risk of developing CVD and diabetes. In addition to the primary outcome of the proportion of patients achieving adequate BP control at 24 and 48 weeks, it will assess secondary outcomes, including a cost-benefit analysis and psychological determinants of adherence and compliance with drug therapy. ${ }^{34}$

\section{Future considerations for telehealth in hypertension}

In the last decade, an increasing number of studies have shown supportive evidence for the use of telehealth in patients with hypertension and other comorbidities. A structured monitoring pathway (short- versus longer-term) as opposed to the unspecified intention of the studies to date will help to answer questions of how best to deploy this technologically and use telehealth possibly in a more strategic fashion. For example, when a patient achieves target BP, they could/should be encouraged to adhere to lifestyle changes and therapies to maintain this, rather than focusing on the monitoring process. Further, monitoring could be used on an interim basis for specific clinical or therapeutic reasons in certain groups.

There are concerns that we have cited in this review that suggest the change in responsibility for measurement and isolation from face-to-face professional support might have a negative impact on the patient's quality of life. There are very little data on the costs versus benefits of this technology and how it relates to the patient's quality of life, which can be addressed with more studies using mixed (quantitative and qualitative) method designs. The monitoring period per se could be investigated as a management utility, and the type of system (fixed, mobile, wireless) or technology for a specific clinical requirement that also meets the patient's needs requires further evaluation. HBPT with self-care versus HBPT with health care professional support needs to be evaluated in a head-to-head, sufficiently powered, randomized controlled study. These studies are important for the development and future adoption of this technology.

\section{Disclosure}

The authors have no conflicts of interest to report.

\section{References}

1. Roger VL, Go AS, Lloyd-Jones DM, et al. Heart disease and stroke statistics - 2012 update: a report from the American Heart Association. Circulation. 2012;125:e2-e220.

2. Joint Health Surveys Unit. Health Survey for England 2011: Health, Social Care and Lifestyles - Summary of Key Findings. London: Health and Social Care Information Centre; 2012. Available from: https:// catalogue.ic.nhs.uk/publications/public-health/surveys/heal-surv-eng2011/HSE2011-Sum-bklet.pdf. Accessed July 24, 2013.

3. Porta M, Sjoelie AK, Chaturverdi N, et al. Risk factors for the progression to proliferative retinopathy in the EURODIAB Prospective Complications Study. Diabetologia. 2001;44:2203-2209.

4. Martínez-Maldonado M. Role of hypertension in the progression of chronic renal disease. Nephrol Dial Transplant. 2001;16 Suppl 1: 63-66.

5. Stevens PE, O’Donoghue DJ, de Lusignan S, et al. Chronic kidney disease management in the United Kingdom: NEOERICA project results. Kidney Int. 2007;72:92-99.

6. Flegal KM, Carroll MD, Ogden CL, Curtin LR. Prevalence and trends in obesity among US adults, 1999-2008. JAMA. 2010;303: 235-241.

7. Krause T, Lovibond K, Caulfield M, McCormack T, Williams B. Management of hypertension: summary of NICE guidance. BMJ. 2011;343:d4891.

8. Zhao W, Katzmarzyk PT, Horswell R, et al. Aggressive blood pressure control increases coronary heart disease risk among diabetic patients. Epub June 11, 2013.

9. Blood Pressure Lowering Treatment Trialists' Collaboration, Turnbull F, Neal B, et al. Effects of different regimens to lower blood pressure on major cardiovascular events in older and younger adults: meta-analysis of randomised trials. BMJ. 2008;336:1121-1123.

10. Go AS, Chertow GM, Fan D, McCulloch CE, Hsu CY. Chronic kidney disease and the risks of death, cardiovascular events, and hospitalization. N Engl J Med. 2004;351:1296-1305. 
11. Parving HH, Lehnert H, Bröchner-Mortensen J, Gomis R, Andersen S, Arner P. The effect of irbesartan on the development of diabetic nephropathy in patients with type 2 diabetes. $N$ Engl J Med. 2001;345: 870-878.

12. Collins R, MacMahon S. Blood pressure, antihypertensive drug treatment and the risks of stroke and of coronary heart disease. $\mathrm{Br} \mathrm{Med}$ Bull. 1994;50:272-298.

13. Booth J. A short history of blood pressure measurement. Proc R Soc Med. 1977;70:793-799.

14. White J, Schick J. Home blood pressure monitoring and diabetes. Clin Diabetes. 2004;22:28-31.

15. Ma Y, Temprosa M, Fowler S, et al. Evaluating the accuracy of an aneroid sphygmomanometer in a clinical trial setting. Am J Hypertens. 2009;22:263-266.

16. Pickering TG. Principles and techniques of blood pressure monitoring. Cardiol Clin. 2002;20:207-223.

17. Omboni S, Gazzola T, Carabelli G, Parati G. Clinical usefulness and cost effectiveness of home blood pressure telemonitoring: meta-analysis of randomized controlled studies. J Hypertens. 2013;31:455-467.

18. Sutton DR, Taylor P, Earle K. Evaluation of PROforma as a language for implementing medical guidelines in a practical context. $B M C$ Med Inform Decis Mak. 2006;6:20.

19. Earle KA, Squires R, Anandarajah S, Taylor P, Sutton D. Evaluation of artificial intelligence and clinical decision making in the management of patients with type 2 diabetes and uncontrolled hypertension. Presented at: 43rd European Association for the Study of Diabetes annual meeting; September 18-21, 2007; Amsterdam, the Netherlands.

20. Omboni S, Guarda A. Impact of home blood pressure telemonitoring and blood pressure control: a meta-analysis of randomized controlled studies. Am J Hypertens. 2011;24:989-998.

21. McCant F, Mckoy G, Grubber J. Feasibility of blood pressure telemonitoring in patients with poor blood pressure control. J Telemed Telecare. 2009; 15:281-285.

22. Crowley MJ, Smith VA, Olsen MK, et al. Treatment intensification in a hypertension telemanagement trial: clinical inertia or good clinical judgment? Hypertension. 2011;58:552-558.

23. Beney J, Bero LA, Bond C. Expanding the role of outpatient pharmacists: effects on health services utilisation, costs, and patient outcomes. Cochrane Database Syst Rev. 2000;3:CD000336.

24. Fahey T, Schroeder K, Ebrahim S. Interventions used to improve blood pressure in patients with hypertension. Cochrane Database Syst Rev. 2006;4:CD005182.
25. Green BB, Cook AJ, Ralston JD, et al. Effectiveness of home blood pressure monitoring, web communication, and pharmacist care on hypertension control: a randomized controlled trial. JAMA. 2008;299: 2857-2867.

26. Rosenstock I, Strecher V, Becker M. Social learning theory and the health belief model. Health Educ Behav. 1988;15:175-183.

27. McManus R, Mant J, Bray EP, et al. Telemonitoring and self-management in the control of hypertension (TASMINH2): a randomised controlled trial. Lancet. 2010;376:163-172.

28. Logan A, Irvine M, McIsaac W, et al. Effect of home blood pressure telemonitoring with self-care support on uncontrolled systolic hypertension in diabetics. Hypertension. 2012;60:51-57.

29. Cartwright M, Hirani SP, Rixon L, et al. Effect of telehealth on quality of life and psychological outcomes over 12 months (Whole Systems Demonstrator telehealth questionnaire study): nested study of patient reported outcomes in a pragmatic, cluster randomised controlled trial. BMJ. 2013;346:f653.

30. Kjeldsen S, Hedner T, Jamerson K, et al. Hypertension optimal treatment (HOT) study. Home blood pressure in treated hypertensive subjects. Hypertension. 1998;31:1014-1020.

31. Earle KA, Istepanian RS, Zitouni K, Sungoor A, Tang B. Mobile telemonitoring for achieving tighter targets of blood pressure control in patients with complicated diabetes: a pilot study. Diabetes Technol Ther. 2010;12:575-579.

32. Shea S, Weinstock RS, Teresi JA, et al. A randomized trial comparing telemedicine case management with usual care in older, ethnically diverse, medically underserved patients with diabetes mellitus: 5 year results of the IDEATel study. J Am Med Inform Assoc. 2009;16(4): 446-456.

33. Egede LE, Strom JL, Fernandes J, Knapp RG, Rojugbokan A. Effectiveness of technology assisted case management in low income adults with type 2 diabetes (TACM-DM): study protocol for a randomized controlled trial. Trials. 2011;12:231.

34. Parati G, Omboni S, Compare A, et al. Blood pressure control and treatment adherence in hypertensive patients with metabolic syndrome: protocol of a randomized controlled study based on home blood pressure telemonitoring vs conventional management and assessment of psychological determinants of adherence (TELEBPMET study). Trials. 2013;14:22.

35. Istepanian RS, Zitouni K, Harry D, et al. Evaluation of a mobile phone telemonitoring system for glycaemic control in patients with diabetes. J Telemed Telecare. 2009;15(3):125-128.
Vascular Health and Risk Management

\section{Publish your work in this journal}

Vascular Health and Risk Management is an international, peerreviewed journal of therapeutics and risk management, focusing on concise rapid reporting of clinical studies on the processes involved in the maintenance of vascular health; the monitoring, prevention and treatment of vascular disease and its sequelae; and the involvement of
Dovepress

metabolic disorders, particularly diabetes. This journal is indexed on PubMed Central and MedLine. The manuscript management system is completely online and includes a very quick and fair peer-review system, which is all easy to use. Visit http://www.dovepress.com/ testimonials.php to read real quotes from published authors. 